\title{
Truncated post-Newtonian Neutron Star Model
}

\author{
Hisa-aki Shinkai团 \\ Department of Physics, Washington University, St. Louis, Missouri 63130-4899, USA \\ (May 15, 1998 / revised June 27, 1998 / accepted for publication in PRD. (Brief Report)
}

\begin{abstract}
As a preliminary step towards simulating binary neutron star coalescing problem, we test a post-Newtonian approach by constructing a single neutron star model. We expand the TolmanOppenheimer-Volkov equation of hydrostatic equilibrium by the power of $c^{-2}$, where $c$ is the speed of light, and truncate at the various order. We solve the system using the polytropic equation of state with index $\Gamma=5 / 3,2$ and 3 , and show how this approximation converges together with mass-radius relations. Next, we solve the Hamiltonian constraint equation with these density profiles as trial functions, and examine the differences in the final metric. We conclude the second 'post-Newtonian' approximation is close enough to describe general relativistic single star. The result of this report will be useful for further binary studies.
\end{abstract}

PACS number(s): 04.25.Dm, 04.25.Nx, and 04.40.Dg

\section{INTRODUCTION}

Several earth-based interferometers designed to detect gravitational waves have been recently constructed. Detectors such as LIGO, VIRGO, GEO and TAMA are expected to begin operating within a few years (see e.g. [i]). In order to extract gravitational waveforms from noisy data and to discuss physical parameters, it is essential to predict waveforms in advance by both analytical and numerical approaches.

Binary neutron star systems are one of the most plausible sources of gravitational waves. They emit energy through gravitational radiation, shrink their inspiral orbits gradually, and finally merge with strong emission of gravitational waves. The system is described by the post-Newtonian ( $\mathrm{PN}$ ) approximation (see e.g. [2]) in the last several minutes before they merge, while in the last phase of coalescence of stars we need to solve the Einstein equations which are available only through numerical integration.

After the pioneering numerical works by Nakamura and Oohara in the Newtonian gravity with radiation reaction correction [3], several groups started developing their numerical codes to solve this problem in a more realistic way. Such hydrodynamical simulations are categorized as in the Newtonian scheme (with/without radiation reaction term) [4 11]; PN approximation [12]; and fully general relativistic level 13,15$]$. However, we do not have a method to construct physically satisfactory initial data for inspiral binaries in general relativity. Most of the numerical tests start their simulations under assumptions of certain quasi-equilibrium and conformal flatness of spacetime, with a particular choice of vorticity of fluid (e.g, 16] and references therein).

One way to prepare initial data might be patching the $\mathrm{PN}$ scheme to the general relativistic one [17]. In this report, we construct a simple model and examine how this effort is justified. We solve the Tolman-Oppenheimer-
Volkov (TOV) equation of hydrostatic equilibrium of a single neutron star, which is truncated at the various PN levels. We compare the mass and radius of a star as a function of central density using the polytropic equation of state. We also solve the Hamiltonian constraint equation of the Einstein equations by substituting these density profiles as trial functions, and discuss the differences in the metric.

This study is an extended one from the earlier works 1821 at the first PN approximation. We intend to make a bridge between the Newtonian and general relativistic solutions of a neutron star model, both of which are first shown numerically by Tooper [22].

In the actual calculations, we used the geometrical units of $c=G=M_{\odot}=1$, where $c, G, M_{\odot}$ are the speed of light, Newton's gravitational constant and the solar mass, respectively. However, $c$ and $G$ will appear in the text where they help understanding.

\section{TRUNCATED TOV NEUTRON STARS}

In general relativity (GR), we have the TOV equation for solving a hydrostatic equilibrium star in the spherically symmetric spacetime. We start from the metric

$$
d s^{2}=-e^{2 \Phi(r)} d t^{2}+e^{2 \Lambda(r)} d r^{2}+r^{2}\left(d \theta^{2}+\sin ^{2} \theta d \varphi^{2}\right),
$$

where $e^{2 \Lambda(r)}=\left(1-\frac{2 G m(r)}{c^{2} r}\right)^{-1}$. Then the TOV equations are written as

$$
\begin{aligned}
\frac{d m}{d r} & =4 \pi r^{2} \rho_{t}, \\
\frac{d p}{d r} & =-\frac{G m \rho_{t}}{r^{2}}\left(1+\frac{p}{\rho_{t} c^{2}}\right)\left(1+\frac{4 \pi p r^{3}}{m c^{2}}\right)\left(1-\frac{2 G m}{r c^{2}}\right)^{-1}, \\
\frac{d \Phi}{d r} & =-\frac{1}{\rho_{t}} \frac{d p}{d r}\left(1+\frac{p}{\rho_{t} c^{2}}\right)^{-1},
\end{aligned}
$$


together with the specified equation of state, for which we use the polytropic equation of state

$$
p=K \rho^{\Gamma}=K \rho^{1+1 / n},
$$

where $p, \rho$ are the pressure and energy density, respectively, and $\rho_{t}$ is the total mass density,

$$
\rho_{t}=\rho+\frac{p}{(\Gamma-1) c^{2}}
$$

Obviously, the set of equations recover the Newtonian limit for $c^{2} \rightarrow \infty$.

The idea of this report is to expand the product of the parentheses in (2.3) and (2.4) and truncate them at the order of $1 / c^{2 i}$. The $i$-th truncation, then, gives the so-called $i$-th $\mathrm{PN}$ approximation. (The case of $i=1$ is briefly mentioned in 23].) That is, we write (2.3) and (2.4) schematically

$$
\begin{aligned}
\frac{d p}{d r}= & -\frac{G m \rho_{t}}{r^{2}}(1+A)(1+B)(1-C)^{-1} \\
= & -\frac{G m \rho_{t}}{r^{2}}(1+A+B+C \\
& \left.\quad+A B+A C+B C+C^{2}+\cdots\right) \\
\frac{d \Phi}{d r}= & -\frac{1}{\rho_{t}} \frac{d p}{d r}(1+A)^{-1} \\
= & -\frac{1}{\rho_{t}} \frac{d p}{d r}\left(1-A+A^{2}-A^{3}+\cdots\right) .
\end{aligned}
$$

If we use these equations with terms in the RHS of up to two products of $A, B, C$ (such as $A B$ or $A^{2}$ ), then we say the system is in the second $\mathrm{PN}$ approximation.

We apply $\Gamma=5 / 3,2$ and 3 for the equation of state ( $n=1.5,1$ and 0.5 in the polytropic index, respectively) and compare the solutions of Newtonian, GR and up to third PN approximation.

The radius of the star, $R$, is measured at the point, $r_{\star}$, where density $\rho_{t}$ drops low enough $\left[O\left(10^{-10}\right)\right.$ in the geometrical units], and given by the proper length,

$$
R=\int_{0}^{r_{\star}}\left(1-\frac{2 G m(r)}{c^{2} r}\right)^{-1 / 2} d r
$$

with appropriate truncation in the integrand. We express the mass of the star, $M$, by $M=m\left(r_{\star}\right)$.

We use 5th order Runge-Kutta method (Fehlberg method) to integrate the equations. In order to check that this approach is right, we also worked the TOV equations in the harmonic gauge and confirmed that we get the identical physical quantities in the results.

In Fig.1, we show the total mass $M$ as the function of the central density $\rho_{c}$ for the different $\Gamma \mathrm{s}$ and PN levels. Mass is in the unit of $M_{\odot}$ and central density is in $\left[\mathrm{g} / \mathrm{cm}^{3}\right]$, and both are rescalable with the constant $K$ in the equation of state. Here we use $K$ in the calculations as: $K_{5 / 3}=4.35$ (for $\Gamma=5 / 3$ ), $K_{2}=10^{2}$ (for $\Gamma=2$ ), and $K_{3}=10^{5}$ (for $\Gamma=3$ ) in the geometrical unit, where $K_{5 / 3}$ is the number for the pure neutron equation of state [24].
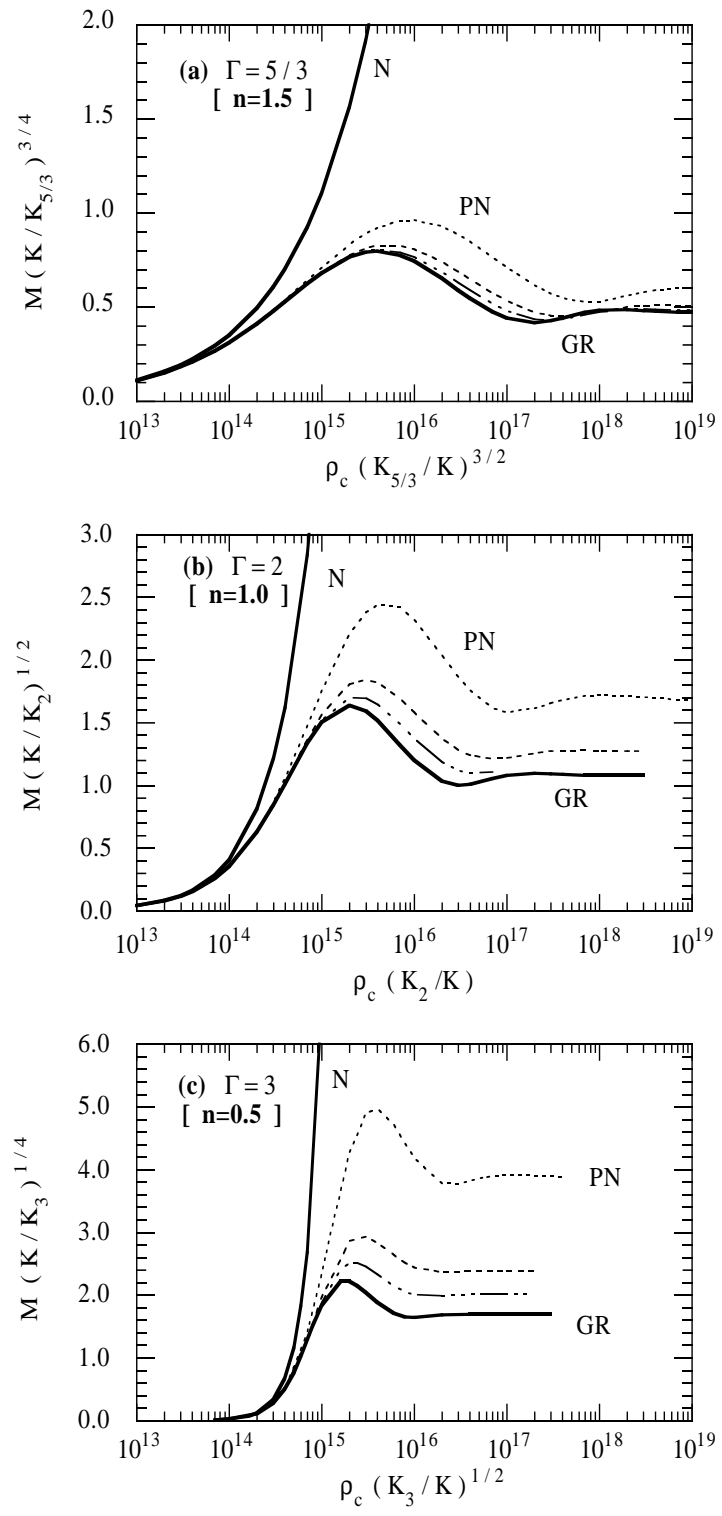

FIG. 1. Total mass as the function of its central density for truncated neutron star model. Figures (a), (b) and (c) are for different equation of state with $\Gamma=5 / 3,2$ and 3 , respectively. Mass is in the unit of solar mass and central density is in $\left[\mathrm{g} / \mathrm{cm}^{3}\right]$. The gray solid line is of Newtonian solutions, the solid line is of general relativistic solution. The dotted line, dashed line and three-dot-line are of first, second and third post-Newtonian approximated solution, respectively. 
We see clearly the convergence of this PN approximation in all the $\Gamma$ s. However, if the equation of state is stiff, then the high density configuration differs from that of GR even at the higher PN approximation.

From the first PN approximation, we see the existence of the maximum mass. The central density which gives this maximum becomes larger in the weak gravity approximation.

In Fig.2, we show the mass-radius relations. In the Newtonian limit, the asymptotic behaviors of $M$ near $M=0$ are as $M \propto R^{-3}$ (for $\Gamma=5 / 3$ ), $M \propto R^{0}$ (for $\Gamma=2$ ) and $M \propto R^{5}$ (for $\Gamma=3$ ). These represent softness (for $\Gamma=5 / 3$ ) and stiffness (for $\Gamma=3$ ) of the equation of state. We see that all the lines in Fig. 2 coincide with this Newtonian limit in the lower mass limit. The figure also shows us that the first PN solution has the same feature as GR.

We also checked the causality constraint $d p / d \rho \leq 1$ (see e.g. 25]) in all of the models, and confirmed that the constraint is always valid.

\section{METRIC OUTPUT VIA HAMILTONIAN CONSTRAINT}

We next solve the Hamiltonian constraint equation in GR with the trial density profiles obtained above. Our aim is to compare the difference of the output metric and to examine a matching scheme of $\mathrm{PN}$ data to the general relativistic one.

We use O'Murchadha-York's conformal approach 26] to solve the Hamiltonian constraint. Defining the conformal factor $\psi$ and setting $\gamma_{i j}=\psi^{4} \hat{\gamma}_{i j}$, the constraint becomes

$$
{ }^{{ }^{(3)}} \hat{\Delta} \psi={ }^{(3)} \hat{R} \psi-16 \pi G \hat{\rho} \psi^{-3}
$$

where ${ }^{(3)} \hat{\Delta}$ and ${ }^{(3)} \hat{R}$ are the 3 -dimensional Laplacian and Ricci scalar curvature, respectively, defined by $\hat{\gamma}_{i j}$. Here we assumed $K_{i j}=\hat{K}_{i j}=0$.

We choose our trial metric $\hat{\gamma}_{i j}$ as conformally flat, and solve (3.1) with a trial density configurations of $\hat{\rho}=\rho_{t}$. We use the Incomplete Cholesky conjugate gradient (ICCG) method [27] with the Robin boundary condition $\psi=1+C / r$, where $C$ is a constant, for solving (3.1).

In Fig.3, we show the conformal factor $\psi$ at the origin as a function of central density of trial configuration. The 3 -metric at the center will be given by $\gamma_{i j}=\psi^{4} \delta_{i j}$. We see that using the Newotnian configuration as input gives us quite different solutions from the expected ones of GR, while all PN trials give similar solutions with GR. Independently to $\Gamma$, we can say second $\mathrm{PN}$ approximation provides closer values for the output metric to those of GR.
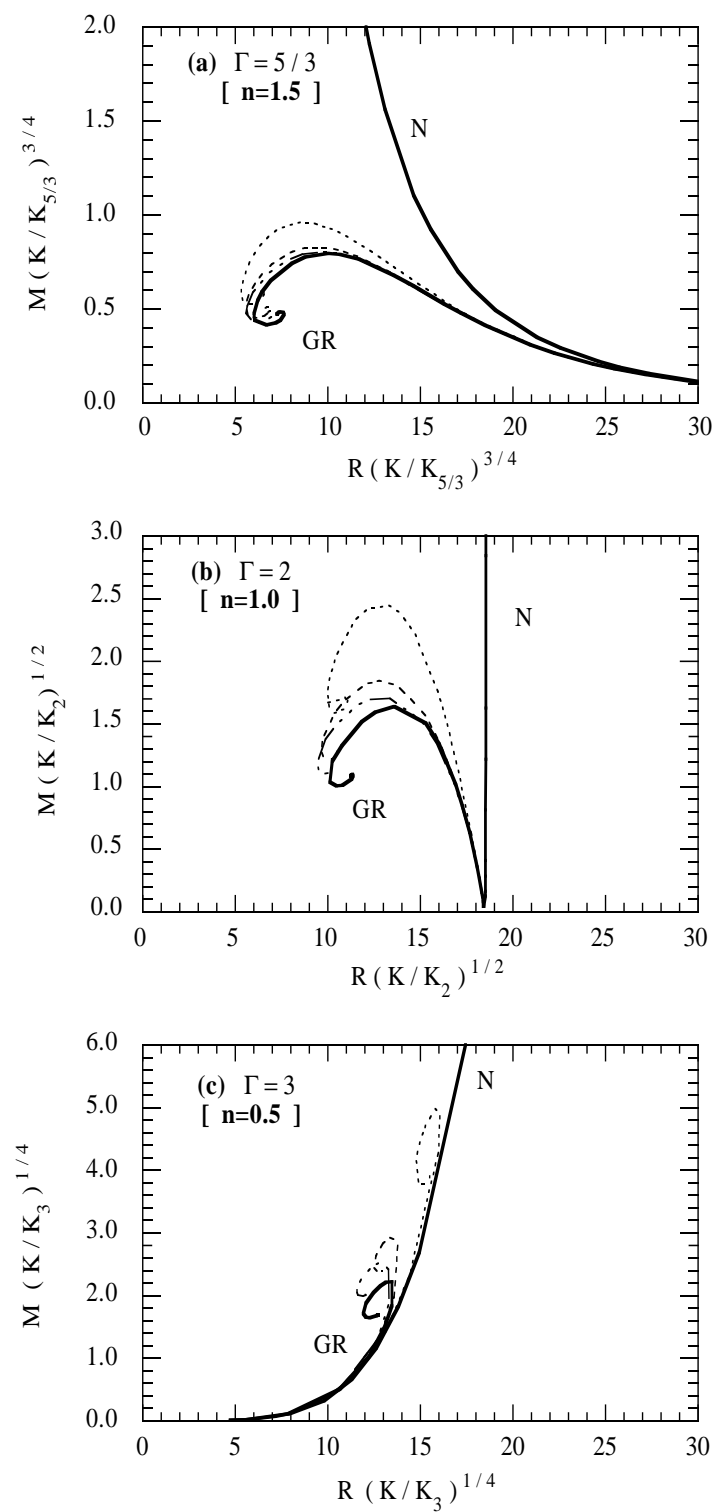

FIG. 2. Mass and radius relations for truncated neutron star models. Mass is in the unit of solar mass and radius is in $[\mathrm{km}]$. The lines are the same as of Fig.1. 


\section{DISCUSSION}
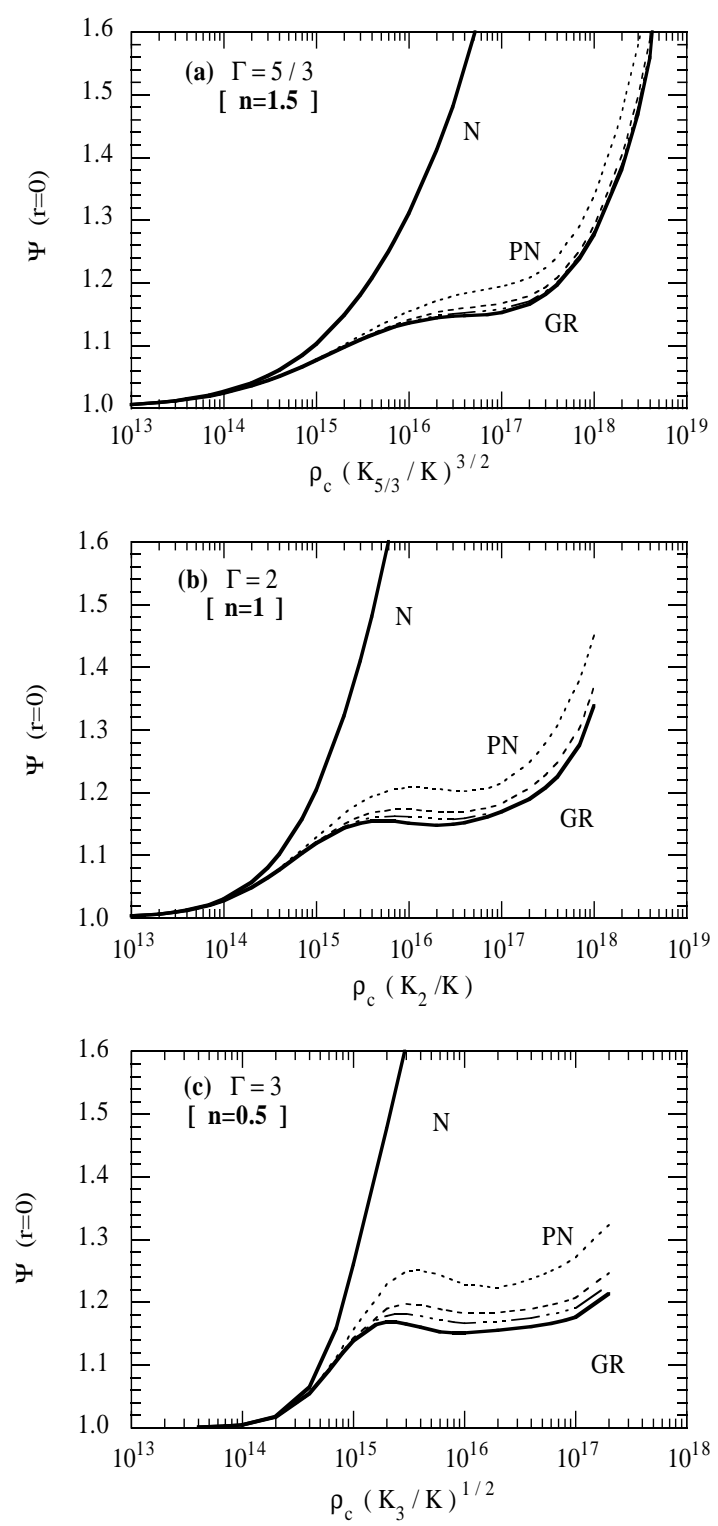

FIG. 3. The conformal factor $\psi$ at the origin is displayed as a function of central density, of which we used a trial configuration for solving Hamiltonian constraint equation. The central density is in the unit of $\left[\mathrm{g} / \mathrm{cm}^{3}\right]$. Each line indicates the trial profile as input, using the same notation with Fig. 1.
In order to justify the recent post-Newtonian (PN) approaches to the binary neutron star problem, we constructed a simple model. By solving the hydrostatic equilibrium equation of a star at $i$-th $\mathrm{PN}$ approximation, we showed the convergence of this approach, the mass and radius relations and resultant metric output via the Hamiltonian constraint equation.

We conclude that second PN approximation provides quite similar density profiles to those of GR, independent of equations of states. If we use second PN density configurations as trial functions, we get closer metric solutions to those from GR through the Hamiltonian constraint. Although this study is restricted to a hydrostatic single star model, we think that the figures shown here are convenient templates for further numerical studies.

As shown in [17], the discontinuous matching surface of PN and GR in the vacuum region will be smoothed out in fully relativistic evolution in a particular slicing condition. Therefore we expect that higher PN initial data will smoothly evolve in the fully relativistic simulations, although there are many unknown factors as to whether such an initial data is numerically satisfactory or not. We are now applying this approach to construct a binary model including their velocity corrections together with fully general relativistic hydrodynamical evolutions. This effort will be reported elsewhere.

Acknowledgments The author thanks Stephen B. Selipsky, Wai-Mo Suen and Cliff. M. Will for discussions. He also thank Ed Seidel and Doug Swesty for the comments on the causality constraint. He appreciates the annonymous referee's suggestions in the section IV. This work was partially supported by NSF PHYS 96-00049, 96-00507, and NASA NCCS 5-153.

¥ Electronic address: shinkai@wurel.wustl.edu

[1] K. S. Thorne, in Black Holes and Relativistic Stars, Proceedings of a Conference in Memory of S. Chandrasekhar, ed. by R. M. Wald (University of Chicago Press, 1998).

[2] C. M. Will, in Relativistic Cosmology ed. by M. Sasaki (Universal Academy Press, 1994). (gr-qc/9403033).

[3] K. Oohara and T. Nakamura, Prog. Theor. Phys. 82, 535 (1989) ; ibid. 82, 1066 (1989) ; ibid. 83, 906 (1990); ibid. 86, 73 (1991).

[4] M. Shibata, T. Nakamura and K. Oohara, Prog. Theor. Phys. 88, 1079 (1992); ibid. 89, 809 (1993).

[5] F.A. Rasio and S.L. Shapiro, Astrophys. J. 432, 242 (1994); ibid. 438, 887 (1995).

[6] J.M. Centrella and S.L.W. McMillan, Astrophys. J. 416, 719 (1993); X. Zhuge, J.M. Centrella and S.L.W. McMillan, Phys. Rev. D50, 6247 (1994); ibid. D54, 7261 (1996). 
[7] M.B. Davis, W. Benz, T.Piran, and F.K. Thielemann, Astrophys. J. 431, 742 (1994).

[8] M. Ruffert, T.-Th. Janka and G. Schäfer, Astron. Astrophys. 311, 532 (1996); M. Ruffert, T.-Th. Janka, K. Takahashi and G. Schäfer, ibid. 319, 122 (1997); M. Ruffert, M. Rampp, H.-Th. Janka, ibid. 321, 991 (1997).

[9] K.C.B. New and J.E. Tohline, Astrophys. J. 490, 311 (1997).

[10] E.Y.M. Wang, F.D. Swesty, and A.C. Calder, astro$\mathrm{ph} / 9806019$ and astro-ph/9806022.

[11] K. Uryu and Y. Eriguchi, Mon. Not. Roy. Astron. Soc. 296, L1 (1998).

[12] K. Oohara and T. Nakamura, Prog. Theor. Phys. 88, 307 (1992); M. Shibata, K. Oohara and T. Nakamura, ibid. 98, 1081 (1997); M. Shibata, T. W. Baumgarte and S. L. Shapiro, gr-qc/9805026.

[13] J.R. Wilson and G.J. Mathews, Phys. Rev. Lett. 75, 4161 (1995); J.R. Wilson, G.J. Mathews and P. Marronetti, Phys. Rev. D54, 1317 (1996); J.R. Wilson and G.J. Mathews, Astrophys. J. 482, 929 (1997).

[14] K. Oohara, T. Nakamura and M. Shibata, Prog. Theor. Phys. Suppl. 128, 183 (1997).

[15] WashU/Potsdam numerical relativity group, in preparation.

[16] S. Bonazzola, E. Gourgoulhon, J.-A. Marck, Phys. Rev. D56, 7740 (1997); E. Gourgoulhon, gr-qc/9804054.

[17] H. Shinkai, W-M. Suen, F.D. Swesty, M. Tobias, E.Y.M. Wang and C. Will, in the proceedings of the 8th Marcel Grossmann Meeting (World Scientific, to be published). gr-qc/9710073

[18] R. V. Wagoner and R. C. Malone, Astrophys. J. 189, L75 (1974).

[19] I. Ciufolini and R. Ruffini, Astron. Astrophys. 97, L12 (1981); Astrophys. J. 275, 867 (1983).

[20] M. A. Castagnino and C. O. Lousto, Nuovo Cimento 99B, 123 (1987).

[21] J. C. Lombardi, Jr., F. A. Rasio and S. L. Shapiro, Phys. Rev. D56, 3416 (1997).

[22] R. F. Tooper, Astrophys. J. 142, 1541 (1965).

[23] R. Kippenhahn and A. Weigert, Stellar structure and evolution, (Springer-Verlag, 1994).

[24] S. L. Shapiro and S. A. Teukoslky, Black holes, White Dwarfs and Neutron Stars, (Wiley, New York, 1983).

[25] R. Geroch and L. Lindblom, Ann. Phys. 207, 394 (1991).

[26] N. O'Murchadha and J. W. York, Jr., Phys. Rev. D10, 428 (1974).

[27] e.g., G. H. Golub, C. F. Van Loan, Matrix Computations, 3rd Ed., (Johns Hopkins Univ. Press, 1996).

* This paper was accepted for publication in Physical Review D. [access code dsj637]. However, since I was strongly suggested that the contents of this paper should be included as a section in our group's future paper, I gave up the publication. 